\title{
Technical Configuration of Portable/Mobile TV White Space Devices: A Conceptual View
}

\author{
Igor Gepko \\ Ukrainian State Centre of Radio Frequencies, Kiev, Ukraine \\ *Corresponding Author: gepko@ucrf.gov.ua
}

Copyright $(2014$ Horizon Research Publishing All rights reserved.

\begin{abstract}
As is known, to eliminate interference, the frequency reuse approach is followed in Digital TV planning similar to cellular network, avoiding the use of the same channel in two neighboring allotments. There are large areas where a certain group of TV channels are deliberately not used. They are called white spaces in TV spectrum (TVWS). Considering the great economical value of TV spectrum, it was proposed to use TVWS for low-power wireless networking on non-interfering (secondary) basis with the licensed (primary) DTV service. At the same time, restrictions imposed on white space devices (WSDs) to protect primary users should not devaluate spectrum for secondary use. The lack of knowledge about the locations of primary receivers as well as unreliability of estimation of the aggregate interference impact caused by the large number of secondary devices accessing the spectrum are reported to be among the key challenges for the use of TVWS. In our view, parameters for protection of primary system should be based on the determination of minimum separation distance, observance of which at a certain area could be ensured. The required shadow margin as well as multi-user margin will be calculated for such minimum safety range. To reuse spectrum efficiently mobile/portable WSDs should support dynamic power control ability which has to be a key role of sensing and operate with the lower power where TV signal is weak. Except of providing WSDs with a list of available channels, the geolocation database should contain recommended parameters for path loss calculations as well as minimum distances which could be ensured for a certain inhabited locality.
\end{abstract}

Keywords TV White Space; Cognitive Radio; White Space Device; Spectrum Sensing; Geolocation Database; Opportunistic Spectrum Access; Secondary Use; Aggregate Interference; LTE Over TVWS

\section{Introduction: "White" Spectrum and Cognitive Radio}

In result of the Regional Radiocommunication Conference (RRC06) held in Geneva in June 2006, TV bands IV-V (470-862 MHz) were devoted to DVB-T usage and divided into 49 channels each with $8 \mathrm{MHz}$. Region 1 was divided into geographical allotments and into the sets of frequency assignments for each of them. To eliminate interference, the frequency reuse approach is followed in DTV planning similar to cellular network, avoiding the use of the same channel in two neighboring allotments [1]. In this way we always have large areas where a specific channel is deliberately not used. These areas are called white spaces in television spectrum (TV white spaces, or TVWS: that is how they look at the coverage map). DTV allocations are very large compared to the size of cells in mobile communication, usually covering areas of several hundreds of square kilometers. Considering the economical value of TV spectrum due to perfect propagation characteristics with reasonable size of antennas, it was proposed to use this "white" bandwidth for low-power low-range wireless networking on non-interfering (secondary) basis with the licensed (primary) DTV transmissions.

The US Federal Communications Commission was a pioneer in developing the concept of using TVWS. In Europe these tasks are carried out by CEPT Spectrum Management Group and CEPT Spectrum Engineering Working Group (WGFM and WGSE). ETSI Reconfigurable Radio Systems Technical Committee is responsible for standardization efforts. Thus, increased spectral efficiency and great savings of spectrum after the transition to DTV makes a profit not for broadcasting only. Many services and applications could benefit from secondary TVWS use [2], for example:

- Wireless low power networks for hotspots and premises in TV bands, as an alternative to the highly congested industrial, scientific, and medical (ISM) band.

- Regional-area networking, especially suited to providing Internet in areas with poor wireline infrastructure.

- 3G/4G networks extension over TVWS, complementing licensed spectrum usage: in particular in femtocells to minimize interference to own macrocells.

- Short Range Devices, and others.

The exploitation of TVWS requires developing of mechanisms allowing to determine safely which TV channel 
could be occupied by this or that secondary device and what is maximum allowed effective isotropic radiated power (EIRP) that can be used. This is the idea of so-called opportunistic spectrum access where mechanisms, employed by the network of such devices (White Space Devices, WSD) is a feature falling in the more general category of Cognitive Radio.

For these, two principal mechanisms were proposed: spectrum sensing and geolocation [3]. In the first, a node of secondary wireless network is equipped with the receiver which periodically scan TV band searching for locally unused channels and estimating signal level that could be permitted for its transmitter. Distributed sensing is also possible, where several nodes exchange their data to increase sensing accuracy.

Due to static locations, service areas and frequency assignments of licensed DTV transmitters, one can create a database containing a map of TV channels assigned to each location along with permissible power level for corresponding WSDs. These devices determine current locations via built-in GPS receivers thus finding sets of channels with their corresponding permissible power levels in the database to avoid interference with primary users. Geolocation-based spectrum allocation can be applied simultaneously or together with the spectrum sensing.

The above approaches were implemented in a number of standards/specifications [4]. Thus, IEEE 802.22 defines the physical (PHY) and medium access control (MAC) layers of a regional cognitive radio access network. Here channels allocation is centralized and based on geolocation approach, while sensing also used to check if TV signal is present [5]. Among the other we should mention IEEE 802.11af, developed for opportunistic unlicensed operation in TV bands, IEEE $802.16 \mathrm{~h}$ which defines WiMAX adopted to work in the TVWS in a cognitive manner, ECMA-392, IEEE P802.19.1 and cognitive IEEE 802.15.4 Zigbee.

For more deep review on the state-of-the-art of protection of primary users in dynamically varying radio environment as well as summary of previous studies on regulatory rules for TV White Space opportunistic usage, the interested reader is referred to recently published in EURASIP WCN collection of articles devoted to 10 years of Cognitive Radio [6,7] (see http://jwcn.eurasipjournals.com/series/TYC).

\section{Problems of Deploying TVWS Radio Networks: the Protection of Primary Services}

Licensed primary users must be reliably protected against potential secondary interference. The required protection of digital TV receivers is very high: only a few percent of them is allowed not to reach their signal-to interference ratio (SIR) targets. At the same time, restrictions imposed on secondary devices should not lead to a devaluation of the white spectrum blocs to such extent which makes useless opportunistic secondary access to them.
The main source of interference to primary systems considered in the literature is emissions from TVWS users (typically out-of-band) that fall in-band for the licensed receiver. There are also in-band emissions from the WSD that fall outside but close to licensed band and couldn't be filtered properly in the TV receiver. In practice interference is a combination of these two.

There are two principal categories of TVWS devices: fixed and portable WSD. First include base stations (BS) operating from a known, fixed location with effective isotropic radiated power (EIRP) up to $4 \mathrm{~W}$ as well as 100 $\mathrm{mW}$ consumer premise equipments (CPEs) [8]. The second category, personal/portable devices, are allowed a maximum EIRP in a range of $40 \div 100 \mathrm{~mW}$. Devices of both categories can use spectrum sensing and could retrieve a list of available channels from some authorized database.

Planning a BS within the protected contour is simplified by a number of factors:

- channels with the less critical protective ratios can be allocated for more powerful fixed devices;

- fixed WSD antenna can be oriented towards the center of protected contour co-directionally to antennas of nearest TV receivers (so any BS must be located on the edge of a certain concentric figure within that contour);

- the opportunity to implement cross-polarization isolation between the secondary system and TV.

Thus, in future the technical parameters of unpredictable and ubiquitous portable WSDs will probably be the most critical issue for compatibility with the digital TV services.

In the common cognitive-radio approach it is assumed that secondary devices will find free spectrum by sensing the signals of primary users. However, in some publications low efficiency and poor spectrum-utilization performance of sensing is alleged [9]. Among the major reasons of this we should mention:

1. Secondary device may miss to detect TV signals because of buildings or other surrounding obstructions even though TV channel is occupied (so called "hidden node problem"). Using the channel according to wrong sensing result causes severe interference to the DTV reception.

2. Not knowing the location of the primary receiver is the key problem. Detection of the primary (DTV) system transmitter's signal by the secondary receiver does not provide reliable information regarding the propagation path between the secondary transmitter and the TV receiver, nor of the primary user's desired path gain.

3 . There is no reliable way for secondary device to assess the aggregate interference caused by large number of other WSDs accessing the spectrum - consequently the first one cannot determine its own maximum allowed transmission power.

Indeed, not knowing the location of the primary receiver is a great difficulty for secondary use of spectrum due to high interference margin needed for protection of incumbent application. According to [9], scanning has little sense in the presence of geolocation database which could indicate the 
occupancy of TV channels in the relevant geographical area in a much more-reliable way. At the same time, it's unlikely to expect the database containing certain information about the location of TV receivers. The statement was that the secondary reuse of TVWS is impractical unless the secondary user's transmitted powers (and the data rate) are extremely low [9].

Such a conclusion was largely predetermined by the interference scenario taken for analysis. The propagation loss between the primary transmitter and receiver is calculated as

$$
L_{1}=L_{12}+(1-\beta) \cdot X_{1},
$$

where the constant $\beta \in\{0 ; 1\}$ is a measure of the correlation between the observed level of primary signal on the input of secondary (sensing) receiver and the same signal on the TV receiver input [9];

$L_{12}$ is a measured value of the pass loss between the primary transmitter and the sensing receiver;

$X_{1}$ is a random value representing the uncertainty related to the unknown distance between the TV transmitter and TV receiver. In our view, study that have been conducted for two boundary correlation values, for $\beta=0$ (where sensing does not make sense at all) and $\beta=1$ (where $L_{1}$ and $L_{12}$ are related by deterministic way, i.e. the location of primary receiver is definitely known) is not a sufficient one.

Our important assumption for the next will be the existence of some minimal distance from which we start addressing the issue of primary receiver protection. In other words, we assume that in the typical TV antenna position secondary devices cannot approach it by an arbitrarily small distance.

\section{Interference Model and Secondary Access Scenario}

Let us start with the assumption that the portable secondary device must not cause harmful interference to TV receiver starting from some minimal distance which is specific for this settlement. Assume next that the secondary device Tx 2 knows the power $P_{1}$ of the primary transmitter $\mathrm{Tx} 1$, thus can perfectly estimate the path losses $L_{12}$ between them (Fig. 1). DVB-T external antennas assumed to be placed on roofs (usually over the 10 meters heights) and have no directional properties with respect to secondary transmitters. The signal-to-interference ratio (SIR) in the primary receiver $\mathrm{Rx} 1$ is then (in $\mathrm{dB}$ )

$$
\operatorname{SIR}=S_{1}-I_{2}=\left(P_{1}-L_{1}\right)-\left(P_{2}-L_{21}\right)=P_{1}-P_{2}-L_{1}+L_{21},
$$

where $L_{1}=L_{12}+X_{1}$ is the path loss prediction between the transmitter and receiver of the primary system;

$$
L_{21}=\bar{L}(r)-X_{2}=\bar{L}\left(r_{0}\right)+10 n \log \left(r_{1} / r_{0}\right)-X_{2},
$$

(we have used a simple propagation model, where the mean loss in $\mathrm{dB}$ follows the inverse $n$ power law dependence of distance $[9,10])$. A zero-mean Gaussian random value $X_{2}$ has standard deviation $6 \div 10 \mathrm{~dB}$ for TV band (the maximal measured value was $11.8 \mathrm{~dB}$ according to [10]);

$\bar{L}(r)$ is the deterministic propagation path loss between the secondary transmitter and primary receiver, and a log-normal random value $X_{2}$ presents shadow-fading path loss component. Thus,

$S I R=P_{1}-P_{2}-L_{12}+\bar{L}(r)-\left(X_{1}+X_{2}\right)$,

Where from

$$
P_{2}+X=P_{R x 1}+\bar{L}(r)-S I R .
$$

Let $S I R$ be the minimal value of wanted-to-unwanted signal ratio at the primary receiver input, such that a desirable reception quality is achieved at the output. For specified conditions (frequency offset), it will be a co-channel (adjacent channel, etc.) protection ratio. Then $P_{2}$ is the median value of secondary transmitter power which provides required SIR at the primary receiver input. The primary signal value measured at the input of the secondary receiver should be taken as primary signal estimate at the TV receiver input (considering TV antenna gain): $P_{R x 1}=P_{1}-L_{12}$. We assume that the secondary transmitter Tx2 which is the nearest to the TV receiver Rx1 (and which is located to the last one at a distance $r_{1}$ ) is the main interferer. In the following, we'll evaluate the aggregate interference caused by other WSD. According to the Law of Cosines, $d_{11}^{2}=d_{12}^{2}+r_{1}^{2}-2 \cdot d_{12} \cdot r_{1} \cdot \cos \varphi$, where $\varphi \in[0 ; 2 \pi]$. Let's $\Delta=d_{11}-d_{12}$, then

$$
\Delta \cdot\left(d_{11}+d_{12}\right)=r_{1}^{2}-2 \cdot d_{12} \cdot r_{1} \cdot \cos \varphi,
$$

And

$$
\Delta \approx \frac{r_{1}^{2}-2 \cdot d_{12} \cdot r_{1} \cdot \cos \varphi}{2 \cdot d_{12}}=\frac{r^{2}}{2 d}-r \cdot \cos \varphi .
$$

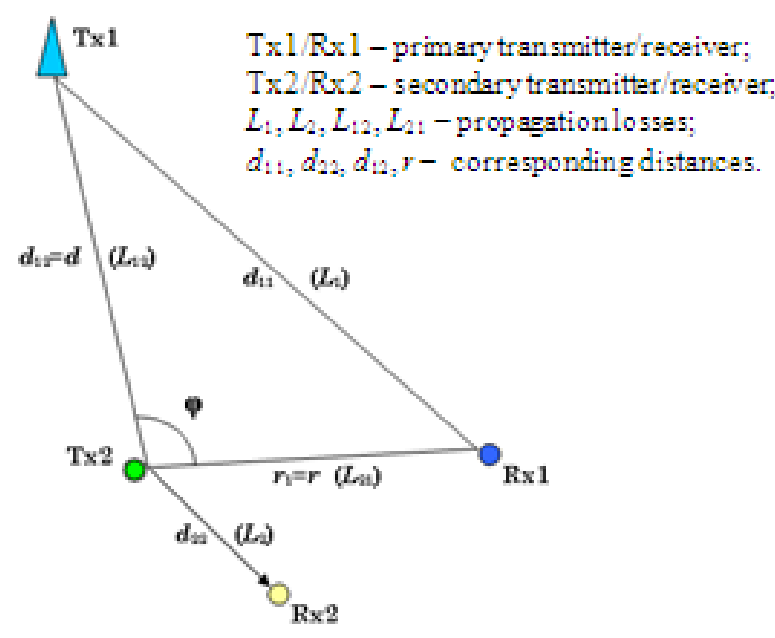

Figure 1. The secondary use and interference scenario. 
It follows $\mathrm{M}[\Delta]=\frac{r^{2}}{2 d}$, and

$$
\begin{gathered}
\sigma_{\Delta}^{2}=\left\langle|\Delta-\mathrm{M}[\Delta]|^{2}\right\rangle= \\
=\mathrm{M}\left[\frac{r^{4}}{4 d^{2}}-2 \frac{r^{2}}{2 d} \cdot r \cdot \cos \varphi+r^{2} \cdot \cos ^{2} \varphi\right]-\frac{r^{4}}{4 d^{2}}=. \\
=\mathrm{M}\left[r^{2} \cdot \cos ^{2} \varphi-\frac{r^{3}}{d} \cdot \cos \varphi\right]=\frac{r^{2}}{2} .
\end{gathered}
$$

Since $X_{1}=10 \cdot n \cdot \log \left(\frac{d+\Delta}{d}\right)$ and $\frac{\Delta}{d}<1$, then we get

$$
X_{1}=\frac{10 \cdot n}{\ln (10)} \cdot\left(\frac{\Delta}{d}-\frac{\Delta^{2}}{2 d^{2}}+\frac{\Delta^{3}}{3 d^{3}}-\frac{\Delta^{4}}{4 d^{4}}+\frac{\Delta^{5}}{5 d^{5}}-\ldots\right)
$$

Taking into account $\sigma_{\Delta}=r / \sqrt{2}, \Delta \approx \mathrm{r}$ (several tens of meters for urban area [8]), and $\mathrm{d} 1 \approx \mathrm{d} 2$ (minimum $0.5 \div 1.5 \mathrm{~km}$ from TV tower, while $15 \div 50 \mathrm{~km}$ is the typical DVB-T coverage range) we conclude $\frac{\Delta}{d} \leq 10^{-2}$. If we take the first term in (2), so that $X_{1}=\frac{10 \cdot n}{\ln (10)} \cdot \frac{\Delta}{d}$, the relative error will unlikely exceed a few percent of X1 actual value. From the aforementioned it follows $\mathrm{m}_{X_{1}}=\frac{10 \cdot n}{\ln (10)} \cdot \frac{r^{2}}{2 d^{2}} \approx 0$, $\sigma_{X_{1}}^{2} \approx\left(\frac{10 \cdot n}{\ln (10)}\right)^{2} \cdot \frac{r^{2}}{2 d^{2}}$ and $\sigma_{X_{1}}=\left(\frac{10 \cdot n}{\sqrt{2} \ln (10)}\right) \cdot \frac{r}{d}$.

Even at extremely large $r$ (hundreds of meters) and the minimal $d$ (a few $\mathrm{km}$ ), the mean square of $X_{1}$ will be about 1 decibel. This value is much smaller in practice. For instance, if $d \approx 5 \mathrm{~km}, r=50 \mathrm{~m}$ and $n=4$, we find $\sigma_{X_{1}} \approx 0.123 \mathrm{~dB}$. Obviously, $X_{1}$ is relatively small compared to $X_{2}$. It follows that the uncertainty of the receiver location is unlikely to be a critical factor for interference scenario which we selected for study.

Since $\Delta$ depends on a number of random factors which are mostly comparable in terms of their impact, the random variable $X_{1}$ has nearly log-normal distribution, and what is much more important, it is small compared to $X_{2}$. In this way, we should consider the variable $X=X_{1}+X_{2}$ to be normally distributed (in $\mathrm{dB}$ ) with the largest possible RMS within $\sigma_{X}=9 \ldots 12 \mathrm{~dB}$.

\section{Basic Relations and Results}

According to (1), the desirable reception quality is achieved for the given conditions and with a given probability Pr. Namely, the right hand side of (1) is a threshold $P_{L}=P_{R x 1}-P R+\bar{L}(r)$ which should not be exceeded in a certain percentage of the time for a some median power $P_{2}$ of the secondary transmitter. Considering normal distribution of $X$ one could write $\operatorname{Pr}\left(P_{2}+X \leq P_{L}\right)=1-Q\left(\frac{P_{L}-P_{2}}{\sigma_{X}}\right) \quad, \quad$ where $Q(z)=\frac{1}{\sqrt{2 \pi}} \int_{z}^{+\infty} \exp \left(-\frac{x^{2}}{2}\right) d x$. For instance, for $95 \%$ of time we have $Q\left(\frac{P_{L}-P_{2}}{\sigma_{X}}\right)=0.05$, or $\frac{P_{L}-P_{2}}{\sigma_{X}}=Q^{-1}=1.65$, where $Q^{-1}$ is the reverse meaning of $Q$-function). From that we have

$$
P_{2}=P_{R x 1}-P R+\bar{L}(r)-Q^{-1} \cdot \sigma_{X}
$$

Knowing the DVB-T antenna gain $G_{A}$, secondary device can estimate the power of TV signal at its input. We could express this as a product of antenna effective aperture and average magnitude of the Poynting vector

$$
P_{R x 1}=\Pi \cdot S_{e f f} \cdot G_{A}=G_{A} \cdot \frac{E^{2}}{120 \pi} \cdot \frac{\lambda^{2}}{4 \pi}=\frac{G_{A}}{480 \pi^{2}} \cdot \lambda^{2} \cdot E^{2}
$$

Being represented in decibel notation (where field strength is expressed in $d B \mu V / m$, power in $\mathrm{dBm}$ and frequency in $\mathrm{MHz}$ ) this formula takes view

$$
P_{R x 1}=E+G_{A}-20 \cdot \log f-10 \cdot \log \frac{160 \pi^{2}}{3}-50 .
$$

Since the DTV receive antenna main lobe is looking away from the omni-directional WSD transmit antenna, we assume $14 \mathrm{~dB}$ off-axis antenna discrimination [8]. Let the minimum nominal distance $r_{0}$ would be $10 \mathrm{~m}$, corresponding to a free space loss of $50 \mathrm{~dB}$ at $800 \mathrm{MHz}$. According to (3), $P_{2}=P_{R x 1}-P R+\bar{L}(r)-Q^{-1} \cdot \sigma_{\mathrm{X}}$. Then considering (1), (4) and [10] we obtain the estimation of the maximum permissible power for the secondary user

$$
P_{2}=E+G_{A}-10\left(\log \frac{160(\pi f)^{2}}{3}-n \log \frac{r}{r_{0}}\right)-P R-Q^{-1} \cdot \sigma_{\mathrm{X}}
$$

This approach [9] is based on assumption that levels of primary signal at both receivers are nearly the same (strongly correlated). It most cases however, primary signal is typically experiencing a greater attenuation at the WSD input than that for fixed TV reception (where antenna location is specifically chosen). This sometimes results in underestimation of permissible power of the secondary transmitter. Indeed, DVB-T reception is usually done with directive roof-top antennas which main lobe is looking away from the nearest omni-directional WSD transmit antenna (14 $\mathrm{dB}$ off-axis antenna discrimination is assumed [8]). But other situation may also happen when secondary device is working somewhere on the upper floors of the building on the opposite side of the street. In this case, the off axis antenna discrimination between the DTV receive antenna and the WSD transmit antenna is not ensured. 
Protection distances needed to protect the primary users for different values of secondary transmitter power (for minimum median field strength $56 \mathrm{~dB} \mu \mathrm{V} / \mathrm{m}$ and DVB-T receiver antenna located at $10 \mathrm{~m}$ height [1]) are presented in the Table 1.

Table 1. Protection distances in meters: WSD/DVB-T (99\%)

\begin{tabular}{|c|c|c|c|c|c|}
\hline \multirow{2}{*}{ channel (PR) } & \multirow{2}{*}{$\mathrm{n}$} & \multicolumn{4}{|c|}{ EIRP of secondary transmitter } \\
\cline { 3 - 6 } & & $40 \mathrm{~mW}$ & $100 \mathrm{~mW}$ & $400 \mathrm{~mW}$ & $1 \mathrm{~W}$ \\
\hline \multirow{2}{*}{$\mathrm{N}(23 \mathrm{~dB})^{*}$} & 3 & 2300 & 3200 & 5000 & 6800 \\
\cline { 2 - 6 } & 4 & 880 & 1120 & 1600 & 2000 \\
\hline \multirow{2}{*}{$\mathrm{N} \pm 1(-30 \mathrm{~dB})$} & 3 & 70 & 90 & 150 & 200 \\
\cline { 2 - 6 } & 4 & 40 & 50 & 75 & 95 \\
\hline \multirow{2}{*}{$\mathrm{N} \pm 2(-42 \mathrm{~dB})$} & 3 & 27 & 36 & 60 & 80 \\
\cline { 2 - 6 } & 4 & 15 & 18 & 27 & 33 \\
\hline
\end{tabular}

* 64-QAM 3/4, fixed roof-level reception

These distances were determined in accordance with (5) for two types of zones: high density urban area $(n=4)$, and medium density urban / suburban environment $(n=3)$ [10]. Protection ratios for co-channel and adjacent channels interference were obtained by averaging the corresponding values for DVB-T/DVB-T2 receivers which provided in [11-14]. We also make the pessimistic assumption that secondary devices operate over the entire $8 \mathrm{MHz}$ bandwidth of TV channel [15].

From the Table 1 it follows that co-channel use requires protection distances from several hundreds of meters up to kilometers even with small power devices. Adjacent channel protection distances for secondary transmitter with power about $40 \mathrm{~mW}$ ranges from 40 up to 70 meters. This distance may also be reduced to 20-27 meters at the expense of less stringent requirements for primary receivers protection $(95 \%$ availability). We assume this to be a minimum nominal separation distance between the DTV receive antenna and the WSD transmit antenna which is required for urban environment. In such circumstances to operate on adjacent channels is allowed for portable WSD which maximum conducted output power does not exceed a few tens of milliwatts (the protection ratio for adjacent channel is assumed to be $-33 \mathrm{~dB}$ ) [8].

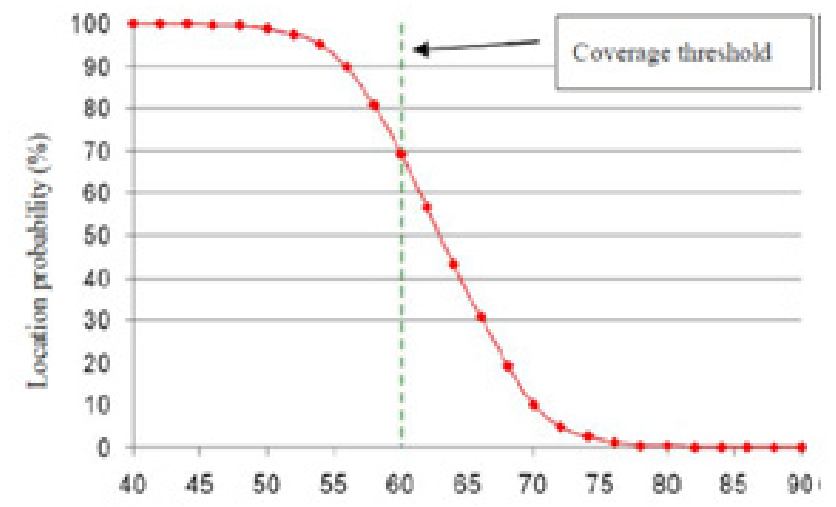

Figure 2. Location probability vs. received field strength (fixed reception) [16].
Minimum median field strength or protected $E_{\min }$ is the lowest field strength value permitting a given standard of reception to be achieved. Therefore, $E_{\min }$ have to be provided at the edge of the coverage area. In practice, the variation range of DVB-T median field strength inside the protected contour is about $50 \mathrm{~dB}$. For a large amount of statistics measurements show a log-normal distribution of the DVB-T signal samples. The measured median value of received field strength is about $63 \mathrm{~dB} \mu \mathrm{V} / \mathrm{m}$ (Fig. 2) [16].

The GE06 Agreement specifies the standard deviation inside large areas as $5.5 \mathrm{~dB}$. Variations may be short-term and noticeable even during the day time period depending on changing weather conditions. Thereby according to (5) permissible power of secondary transmitter will also have a log-normal distribution. Figure 3 shows the probability density of $P_{2}$ for different conditionally named types of environment (where low densities of TV-receivers in industrial districts were also taken into consideration).

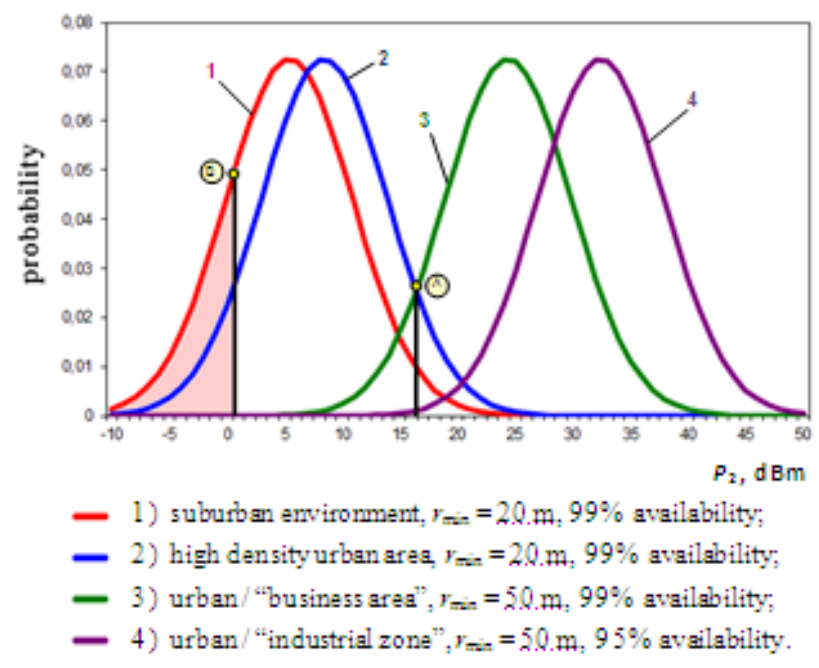

Figure 3. Permissible power of secondary transmitters.

For environments differing by housing density as well as by average building height (such as suburban and urban area), the difference in median permissible power is about $3 \mathrm{~dB}$ at the distance $20 \mathrm{~m}$ between the WSD and TV receiver, and up to $7 \mathrm{~dB}$ at the distance $50 \mathrm{~m}$ (see curves 1 and 2). Reducing from 50 to 20 meters the separation distance between WSD and $\mathrm{TV}$ by $16 \mathrm{~dB}$ decreases the permissible power of secondary transmitter. In contrast with that, reducing the requirements for primary receiver protection (from $99 \%$ to $95 \%$ availability of primary service) nearly for $8 \mathrm{~dB}$ increases the permitted power.

The FCC has allowed portable TVBDs to operate on adjacent channels within the DTV protected contour only if their maximum conducted output power does not exceed 40 $\mathrm{mW}$ [8]. But in densely populated areas with a large number of households (curve 2 in Fig. 3) even WSD with the power of $40 \mathrm{~mW}$ is a potential source of interference for most of receivers: we see that the $16 \mathrm{dBm}$ value exceeds at least $96 \%$ of all power values which could be permitted for a certain location (see "A", Fig. 3). On the other hand, in 
non-residential or sparsely populated areas (for instance hotspots in business center) even being located at a considerable distance from the victim TV receiver $\left(r_{\min }=50\right.$ m: curve 3) the same portable device operates with power $10 \mathrm{~dB}$ less of median permissible value. Obviously, this results to underutilization of available spectrum resource.

Thus, the operation in a fixed power mode is usually far from optimal for WSD. However, the evaluation of $P_{2}$ based on some propagation pattern without sensing is unlikely to provide a much better result. For instance, according to (5) for $E=56 \mathrm{~dB} \mu \mathrm{V} / \mathrm{m}$ we get $P_{2} \approx 0 \mathrm{dBm}$ (see "B", Fig. 3 ). In that case, about $16 \%$ of WSDs operates with power exceeding the recommended level and $84 \%$ with power less of this value. This means the available spectrum resource is underused in terms of coverage and data rate. As it was expected limited knowledge/flexibility regarding the current system parameters leads to large safety margins for secondary-spectrum use and poor spectrum utilization [9].

To use spectrum efficiently, mobile/portable WSDs should support dynamic power control ability. Geolocation approach is unlikely to be an effective solution since the minimum distance between the WSD and the victim TV receiver is much smaller than the typical pixel size $(100 \times 100$ $\mathrm{m} \div 250 \times 250 \mathrm{~m})$. Therefore, measurements made with the special receiver within the pixel are not correlated sufficiently with the primary signal on the input of TV receiver.

In these circumstances sensing made by secondary device seems to be a natural source of information about the local electromagnetic environment. By estimate of the median power of primary signal at the input of potentially nearest TV receiver, WSD evaluates the EIRP of its own transmitter thus implementing Open Loop Power Control scheme in the secondary system.

\section{Evaluation of Aggregated Interference Effect}

Secondary access is commercially attractive only when it is scalable and can support a sufficient amount of secondary traffic in a large area. This means that the primary spectrum is reused by multiple secondary users. However, there are still no unified guidelines for evaluation of the multiple secondary users impact and this issue correspondently has not been properly addressed in the existing regulatory approaches [9].

In the ECC Report 159 a method to regulate transmission powers of secondary users in TV white spaces is proposed [3]. In brief, the main idea is to divide a service area into many pixels. In each of them the maximum allowed transmission power for a secondary user is calculated with the constraint of the TV coverage probability, as follows:

$$
\operatorname{Pr}\left[P X_{P} \geq P X_{P, \min }+I_{T V}+I\left(P_{S, \text { max }}\right)+I M\right] \geq q,
$$

where $q$ is the required TV-coverage probability, $R X_{P}$ is the power of primary signal at the input of TV receiver, $R X_{P \min }$ is the minimum TV-receiver sensitivity, $I_{T V}$ is the interference from other TV transmitters and $I\left(P_{S, \max }\right)$ is the interference from the secondary user as a function of $P_{S, \max }$. The safety margin and multi-user margin for considering the effect of multiple secondary users are accounted for by the term IM. However, a harmonized generally accepted method for obtaining the proper $I M$ value has not yet been adopted [9]. A conservative value of $I M$ results in poor spectrum utilization, while insufficient multi-user margin leads to a risk of failing TV receiver's protection. Nevertheless, we consider this approach to be quite appropriate when there is a dominant source of interference, so that multi-user margin can be determined in relation thereto as a scaling factor.

Let $P_{\mathrm{Rx} 1}^{(1)}=P_{2}-10 n \log r_{1}$ be the median power of signal from the nearest WSD measured at the input of the TV receiver. The interference from other WSDs respectively is $P_{\mathrm{Rx} 1}^{(2)}=P_{2}-10 n \log r_{2} \quad ; \quad \ldots \quad ; \quad P_{\mathrm{Rx} 1}^{(k)}=P_{2}-10 n \log r_{k}$ $\left(P_{\mathrm{Rx} 1}^{(k)}=P_{2} / r_{k}^{n}\right.$ in a linear scale).

Let's consider an example scenario 1, where secondary devices are located at distances $r_{1}=r ; r_{2}=2 r ; \ldots r_{k}=k \cdot r$ from the TV receiver (Fig. 4) so that $P_{\mathrm{Rx} 1}^{(k)}=\frac{1}{k^{n}} \cdot \frac{P_{2}}{r^{n}}=\frac{1}{k^{n}} \cdot P_{\mathrm{Rx} 1}^{(1)}$.

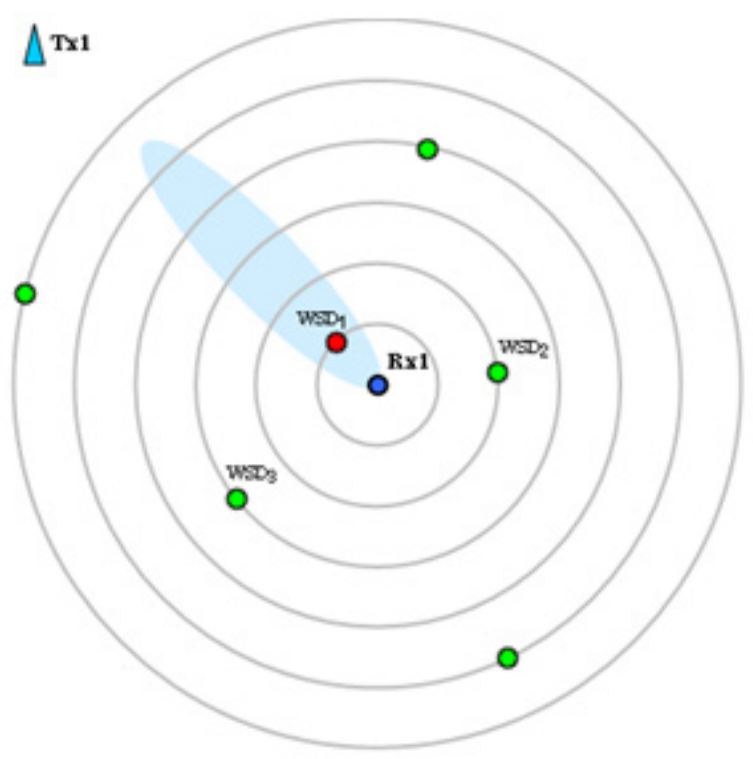

Figure 4. Aggregate interference scenario 1.

Accordingly, the aggregate interference power will be $I_{\Sigma}=\sum_{k=1}^{\infty} P_{\mathrm{Rx} 1}^{(k)}=P_{\mathrm{Rx} 1}^{(1)} \cdot \sum_{k=1}^{\infty} \frac{1}{k^{n}}$. The last sum is known as the Riemann zeta function [17]: $\sum_{k=1}^{\infty} \frac{1}{k^{n}}=1+\frac{1}{2^{n}}+\frac{1}{3^{n}}+\ldots=\zeta(n)$ (where $n>1$ ), thus $I_{\Sigma}=P_{\mathrm{Rx} 1}^{(1)} \cdot \zeta(n)$. According to Euler, the values of the Riemann zeta function at even positive integers 
are expressed in terms of Bernoulli numbers [17] which in their turn can be found from the recurrence relation

$$
\zeta(2 k)=(-1)^{k+1} \frac{(2 \pi)^{2 k}}{2(2 k) !} B_{2 k}, \quad \sum_{j=0}^{k}\left(\begin{array}{c}
k+1 \\
j
\end{array}\right) B_{j}=0, k \geq 1 .
$$

As it follows from (6), $B_{0}=1, B_{1}=-1 / 2$. The value of $\zeta(3)$ is known as Apéry's constant [18], while the value of $\zeta(5)$, which is also one of the widely known mathematical constants, was published in [19]. In this case, a few initial values of $\zeta(n)$ have significance.

A second scenario presents a uniform discrete distribution, with distances between the TV receiver and WSDs relating to each other as a series of natural numbers. The nearest of these devices is at the critically small distance $r_{1}=r$ from the primary receiver (at the minimal distance specified for this location), the other devices - at distances twice (three times, etc.) greater. Thus their amount increases proportionally to the square of the radius from the TV receiver and this asymptotically leads to uniform spatial distribution. The directional properties of the antennas change the contribution of different transmitters in the aggregate interference, but do not affect their total power.

Let $r_{k}^{2}$ be the total number of secondary users within a range of distances $\left[0 ; r_{k}\right]$ from a certain TV receiver (the scenario implies the density $\frac{1}{\pi \cdot r_{1}^{2}}$ of simultaneously active WSDs which is about 127 devices per $1 \mathrm{~km}^{2}$ for $r_{1}=50 \mathrm{~m}$. This much exceeds the density of active IMT users accepted for compatibility studies in the frequency band 790-862 $\mathrm{MHz}$ [20]). One could roughly define their number at distance $\quad r \approx r_{k} \quad$ as $\quad r_{k}^{2}-r_{k-1}^{2}$. It follows $I_{\Sigma}=\sum_{k=1}^{\infty} P_{\mathrm{Rx} 1}^{(k)}=P_{\mathrm{Rx} 1}^{(1)} \cdot \sum_{k=1}^{\infty} \frac{k^{2}-(k-1)^{2}}{k^{n}}$ Since $\sum_{k=1}^{\infty} \frac{k^{2}-(k-1)^{2}}{k^{n}}=\sum_{k=1}^{\infty} \frac{2 k-1}{k^{n}}=2 \cdot \sum_{k=1}^{\infty} \frac{1}{k^{n-1}}-\sum_{k=1}^{\infty} \frac{1}{k^{n}}$, we get $I_{\Sigma}=P_{\mathrm{Rx} 1}^{(1)}(2 \zeta(n-1)-\zeta(n))$. To take into account the impact of aggregate interference an additional "multiuser" safety margin

$I M=2 \zeta(n-1)-\zeta(n)$ about of $3 \mathrm{~dB}$ or even less is needed (see Table 2). This table also shows that this impact depends on the propagation environment. It should not be neglected for slowly attenuating pass loss open areas e.g., rural or suburban. But for medium with the rapid attenuation $(n=4 \div 5)$ this effect is not significant so using multi-user margin may not be necessary.

Table 2. Multiuser margin for different environments

\begin{tabular}{|c|c|c|c|c|}
\hline $\mathrm{n}$ & 3 & 4 & 5 & 6 \\
\hline $2 \zeta(\mathrm{n}-1)-\zeta(\mathrm{n})$ & 2.087818 & 1.321776 & 1.127726 & 1.056496 \\
\hline
\end{tabular}

For this scenario, Table 3 shows the dependence of the allowed WSDs power from the distance between the TV receiver and nearest secondary device (where primary service availability assumed to be $95 \%$ or $99 \%$ ).

Table 3. Maximum allowed transmission power for channel $N \pm 1$

\begin{tabular}{|c|c|c|c|c|c|}
\hline \multirow{2}{*}{ Surroundings } & \multirow{\%}{*}{} & \multicolumn{4}{|c|}{ minimum safety distance between the WSD and } \\
\cline { 2 - 6 } & & $\mathbf{2 0 ~ \mathbf { ~ }}$ & $\mathbf{3 0 ~ \mathbf { ~ }}$ & $\mathbf{4 0 ~ \mathbf { ~ }}$ & $\mathbf{5 0 ~ \mathbf { ~ m }}$ \\
\hline \multirow{3}{*}{ Urban area } & $95 \%$ & $33 \mathrm{~mW}$ & $170 \mathrm{~mW}$ & $0.5 \mathrm{~W}$ & $1.2 \mathrm{~W}$ \\
\cline { 2 - 6 } & $99 \%$ & $5 \mathrm{~mW}$ & $25 \mathrm{~mW}$ & $85 \mathrm{~mW}$ & $200 \mathrm{~mW}$ \\
\hline \multirow{3}{*}{ Suburbs } & $95 \%$ & $33 \mathrm{~mW}$ & $100 \mathrm{~mW}$ & $250 \mathrm{~mW}$ & $450 \mathrm{~mW}$ \\
\cline { 2 - 6 } & $99 \%$ & $8 \mathrm{~mW}$ & $25 \mathrm{~mW}$ & $65 \mathrm{~mW}$ & $130 \mathrm{~mW}$ \\
\hline
\end{tabular}

As the reference medium here we considered high-density $(n=4)$ urban environment with the maximum shadow-fading standard deviation $\sigma=12$ as well as low-rise buildings / suburbs $(n=3, \sigma=9)$. Assuming 20 meters to be a minimum potential distance between the WSD and TV set, we see that aggregate interference impacts the allowed power of WSD in a way that it becomes smaller than that of nominal $40 \mathrm{~mW}$ value [8].

\section{The Technical Configuration of WSDs}

Even in case of mass deployment, for a long period WSDs will unlikely be the cheapest devices at the same time providing locally available, highly localized in time and space services. License-exempt networking will no doubt contribute in reducing the cost of services, but this unlikely to be a compensation of all above shortcomings from the consumer point of view. Sales of WSDs in the form of individual device will hardly allow the manufacturer to benefit from the economies of scale in a reasonable term. That is why the implementation of the WSD functionality as a special module of $3 \mathrm{G} / 4 \mathrm{G}$ user equipment could be considered as the one of the possibilities to ensure its market success (TVWS can particularly be utilized by femtocells for minimizing the interference to macrocells). In such alliance white space device will obtain free GPS capability as well as an independent wireless channel connecting it to geolocation database with the channel tables while smartphone acquires a supplementary frequency band with perfect propagation characteristics.

The key issue to be solved via access to the geolocation database is to provide secondary devices with a consistent list of protected services for given location and locally available channels. The low frequency of changing of this information allows updating it via main portable equipment interface $3 \mathrm{G} / 4 \mathrm{G}$ with the periodicity of a weather forecast or even via USB when recharging from the user's PC. The database could also contain recommended parameters for calculations of the propagation loss (taking into account the actual terrain morphology) as well as nominal minimum safety distances associated with each location (them both 
could gradually be specified in-service after the introduction of cognitive network into operation).

White space devices should operate at variable power levels using the lower power in areas of poor availability of the TV services. The key task of cognitive spectrum sensing has to be power control, where each portable device is responsible for determining of allowed transmission power. The Open Loop Power Control scheme operates based on the estimate of median value of primary signal at the input of WSD receiver and uses the value of minimum recommended distance for current location. Obviously, with this approach the hidden terminal problem will never occur. If there exists a reliable data source (a geolocation database) indicating that the channel is used, a low (or undetectable) level of this channel at the input of WSD informs the last regarding the adverse reception conditions for the TV receiver nearest to it. This permits secondary device to evaluate correctly its maximum allowed transmission power.

Long Term Evolution (LTE) with its advanced power control and flexible deployment in terms of bandwidth and carrier aggregation features looks to be a good choice as the physical layer for implementation of white space devices. At today's radio equipment market we can see LTE devices capable to operate in at least six different bands, including the 791-821/832-862 MHz band. It would be nice if the combined terminals extend LTE operational mode over the TVWS and will operate in the digital dividend I and II bands $(790 \div 862 \mathrm{MHz}$ and $694 \div 790 \mathrm{MHz}$ ) both on the primary as well as secondary basis. After the expected allocation of the digital dividend II to mobile service at the World Radio Conference-2015 an imbalance of needs in the DTV and mobile services in different countries will probably remain. So equipment able to change the operational mode (primary to opportunistic and vice versa) will provide flexibility regarding the coordination procedures in border areas for neighboring countries which follow different priorities in use of spectrum.

The sharing of spectrum is effective if the requirements for the use of spectrum of primary system differ sufficiently from the usage pattern of the secondary system. The secondary systems should also have accurate information about the technical features and usage pattern of the primary system (including location of transmitters, local relief, building characteristics and relevant propagation losses). This is unrealistic with respect to wireless microphones, video cameras and other program making and special events equipment due to liberalized nature of their application. Some administrations see solution in localizing their operation within one or two TV channels. In particular, Ofcom has licensed the channel 38 for exclusive access by wireless microphones and other PMSE equipment. In case it does appear to be not sufficient for large events with several dozen microphones in use, primary users could operate other channels as well. These devices are registered in a database on a temporary basis. WSD periodically interrogates the database to find out which channels are free (updates will be typically every 2 hours) [2].

\section{Conclusion}

The instability of propagation environment and limited accuracy of pass loss predictions makes it reasonable the dynamical setting of margin for primary receiver protection. The study presented in this paper unveils the necessity for revising functions associated with sensing abilities (as well as, to some extent with the geolocation) in cognitive radio system. The geolocation database provides secondary devices with a list of locally available channels as well as with parameters for path loss calculations and minimum safety distance, observance of which at a given location could be ensured. The key role of spectrum sensing is to implement a power control scheme, whereby each portable device evaluates its allowed transmission power. This is based on estimation of the median primary signal value at the input of WSD receiver as well as minimum safety distance for current location.

Parameters for protection of primary system should be based on the determination of minimum separation distance, observance of which at a certain area could be ensured. The multi-user margin is calculated for this minimum safety range as a scaling factor in reference to the dominating interference source. At this approach the uncertainties of TV receivers location will not limit dramatically the accuracy of calculation the WSD power margin needed for TV protection.

Optimization of spectrum sharing implies significant differences in usage patterns of the primary and secondary systems. The last one should have accurate information about the technical features of the primary system, including location of transmitters, local relief and building characteristics. This is unrealistic with respect to PMSE devices due to liberalized nature of their application. Therefore it is advisable ensure their interference-free operation by localizing this equipment within one or two channels) exclusively allocated to them.

We are not expecting the sales of WSDs as individual devices will allow a manufacturers to benefit from the economies of scale in a reasonable term. At the same time, the implementation of WSD as a special module of the $3 \mathrm{G} / 4 \mathrm{G}$ cellular user equipment is an attractive solution for the market. For instance, LTE equipment which is able to operate both on the primary and secondary basis could provide the necessary spectrum use flexibility after the expected allocation of the digital dividend II band to mobile service at the WRC-2015.

\section{REFERENCES}

[1] ITU, "Final Acts of the Regional Radiocommunication Conference for Planning of the Digital Terrestrial Broadcasting Service in Parts of Regions 1 and 3, in the Frequency Bands $174-230 \mathrm{MHz}$ and $470-862 \mathrm{MHz}$ (RRC-06)," Geneva, Switzerland, 2006. 
[2] Fitch, M. et al., "Wireless Service Provision in TV White Space with Cognitive Radio Technology: A Telecom Operator's Perspective and Experience," IEEE Commun. Mag., vol. 49, no. 3, Mar. 2011, pp. 64-73.

[3] CEPT ECC, "ECC Report 159: Technical and Operational Requirements for the Possible Operation of Cognitive Radio Systems in the White Spaces of Frequency Band 470-790 MHz," Jan. 2011. 10. FCC Notice of Proposed Rulemaking," Doc. 12-118, Oct. 2, 2012.

[4] Wang, J., Ghosh, M., Challapali, K., "Emerging Cognitive Radio Applications: A Survey," IEEE Commun. Mag., vol. 49, no. 3, Mar. 2011, pp. 74-81.

[5] IEEE Std 802.22-2011, "IEEE Standard for Information Technology Telecommunications and Information Exchange between Systems Local and Metropolitan Area Networks Specific Requirements - Part 22: Cognitive Wireless RAN Medium Access Control (MAC) and Physical Layer (PHY) specifications: Policies and procedures for operation in the TV Bands," July 2011.

[6] Palicot et al.: "Special issue on 10 years of cognitive radio: state-of-the-art and perspectives," EURASIP Journal on Wireless Communications and Networking 2012 2012:214.

[7] Noguet, D., Gautier, M., Berg, V., “Advances in opportunistic radio technologies for TVWS," EURASIP Journal on Wireless Communications and Networking 2011, 2011:170.

[8] Kang, K.-M. et al., "Deployment and Coverage of Cognitive Radio Networks in TV White Space", IEEE Communications Magazine, Vol. 50, Issue 12, December 2012, pp. 88-94.

[9] Zander, J., Sung, K. W., “Opportunistic Secondary Spectrum Access: Opportunities and Limitations," Radio Science Bulletin, No. 400, March 2012, p. 29 - 33.

[10] Sklar B., "Rayleigh fading channels in mobile digital communication systems part I: characterization", IEEE Communications Magazine, Vol. 35, Issue 7, July 1997, pp. 90-100.
[11] ITU-R Rec. BT.2033, "Planning criteria, including protection ratios, for second generation of digital terrestrial television broadcasting systems in the VHF/UHF bands," 2013.

[12] ETSI EN 300744 V1.6.1 (2009-01). Digital Video Broadcasting (DVB); Framing structure, channel coding and modulation for digital terrestrial television.

[13] J. Kerttula and R. Jäntti, "DVB-T Receiver Performance Measurements Under Secondary System Interference," In Proc. COCORA 2011, Budapest, Hungary, April 2011, pp. 75-80.

[14] Talmola, P. et al., "Field measurements of WSD-DTT protection ratios over outdoor and indoor reference geometries", In Proc. 7th International ICST Conference on Cognitive Radio Oriented Wireless Networks and Communications (CROWNCOM) (June 2012), pp. 7-12.

[15] Makris, D., Gardikis, G., and Kourtis, A., "Quantifying TV White Space Capacity: A Geolocation-Based Approach", IEEE Communications Magazine, Vol. 50, Issue 9, September 2012, pp. 145-152.

[16] ITU-R Rec. SM.1875, "DVB-T coverage measurements and verification of planning criteria," 2010.

[17] Gradshteyn, I.S., Ryzhik, I.M., "Table of Integrals, Series, and Products," translated from Russian, Elsevier Academic Press, USA, 2007.

[18] Lupas, A., "Formulae for some classical constants," in Proceedings of ROGER-2000, Schriftenreihe des Fachbereichs Mathematik, SM-DU-485, Gerhard Mercator Universität Duisburg, 70-76.

[19] Zudilin V.V., "A Third-Order Apéry-Like Recursion for $\zeta(5), "$ Mathematical Notes, Springer, Volume 72, No. 5, 2002, p. $733-737$.

[20] ITU-R Report M.2039-2, "Characteristics of terrestrial IMT-2000 systems for frequency sharing \& interference analyses", 2010. 